\title{
Switch-hitting RNase
}

In the Gram-negative bacterium Escherichia coli, mRNA decay is initiated by RNase E-mediated endonucleolytic cleavage. By

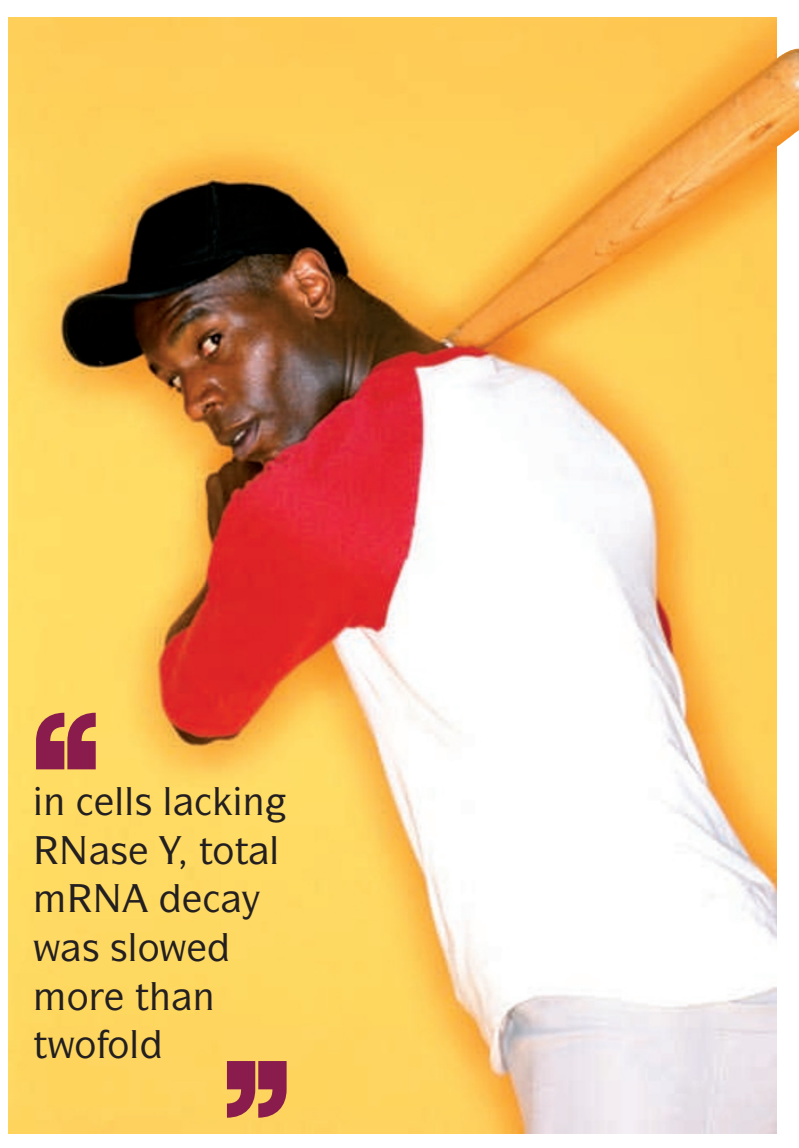

contrast, Gram-positive Bacillus subtilis lacks RNase E but does contain two enzymes, RNase J1 and RNase J2, that have endonucleolytic and $5^{\prime}-3^{\prime}$ exonucleolytic activity and are known to be have roles in the degradation of specific structural mRNAs. However, in strains lacking these two nucleases the half-life of total cellular mRNA is only marginally increased, suggesting a role for an as yet unidentified factor in global mRNA turnover in B. subtilis.

Shahbabian et al. looked at the events involved in degradation of the $S$-adenosylmethionine (SAM)dependent yitJ riboswitch. In these transcripts, the binding of SAM to the untranslated leader region causes a structural rearrangement that prevents the formation of the antiterminator loop structure. This favours the formation of the terminator loop structure, which triggers premature termination of transcription. When cells were starved of methionine, leading to decreased levels of SAM, the full-length yitJ transcript could be detected. However, in the presence of methionine the authors were unable to detect either the full-length transcript or the prematurely terminated transcript, suggesting that there was efficient transcript turnover. Loss of RNase J1 and RNase J2 did not stabilize the terminated transcript. However, when the authors repressed the expression of $y m d A$, which encodes a protein that was recently shown to interact with RNase J1, they were able to detect an RNA species that corresponded to the prematurely terminated $y i t J$ transcript.

In vitro analysis showed that the $y m d A$ gene product was unable to cleave the yitJ leader sequence in the absence of SAM but exhibited endonuclease activity in the presence of SAM, leading the authors to rename this protein RNase Y. Further analysis revealed that cleavage of the yitJ transcript by RNase Y was dependent on $5^{\prime}$ phosphorylation, that cleavage occurred at two distinct sites and that RNase Y was responsible for initiating the degradation of all SAM-dependent riboswitches in B. subtilis.

Interestingly, in cells lacking RNase Y, total mRNA decay was slowed more than twofold, suggesting an important role for this newly identified endonuclease in global mRNA turnover in B. subtilis.

Andrew Jermy

ORIGINAL RESEARCH PAPER Shahbabian, K., Jamalli, A., Zig, L. \& Putzer, H. RNase Y, a novel endoribonuclease, initiates riboswitch turnover in Bacillus subtilis. EMBO J. 24 Sep 2009 (doi:10.1038/emboj.2009.283) 